\title{
La Country, histoire d'une renaissance
}

\section{Abstract :}

Old-time time music is becoming an increasing force within mainstream country music. On the wake of the Coen Brothers' blockbuster, "O Brother", a renewed interest for traditional American music, from folk to bluegrass, is sweeping the country, attracting a younger, more feminine audience. After having mapped out the various territories and eras of classic country music, this paper explores the nostalgic and religious dimensions of the current revival and analyses the changes it triggered in the economics of country music.

Mots clés:

old-time music, country music, folk, hillbilly, O Brother.

Cet article se propose de faire le point sur la fortune actuelle de l'old-time music, composante majeure de ce que par simplification nous appelons country music de ce côté-ci de l'Atlantique. Si le terme country garde en Europe sa valeur générique, il cohabite aux Etats-Unis avec d'autres appellations plus spécifiques, et précisément celle de old-time music, parfois remplacée par mountain music (il faut comprendre ici la chaîne des Appalaches), folksong ou folk music. On tend par ailleurs de plus en plus à regrouper cette tradition avec ses différentes relectures contemporaines sous l'étiquette Americana, terme dont la connotation nationaliste est pourtant bien étrangère à l'esprit d'origine. Mon étude s'appuiera sur des données économiques et sociales complétées par des remarques d'ordre lexicographique. Mais c'est par un balayage à la fois temporel et spatial que nous devons commencer pour situer le contexte dans lequel l'old-time music entame sa nouvelle vie.

Lorsque le potentiel commercial des musiques rurales s'est confirmé, au début des années 1920, et qu'il a fallu les nommer pour en définir la cohérence géographique (le sud), sociale (la campagne) et ethnique (blanche), un producteur yankee de l'époque imposa hillbilly (bouseux, péquenot), du nom d'un quatuor, les Hill-Billies, qui enregistra pour le label Okeh en 19251. Ces musiques se sont développées dans les communautés de colons britanniques, en particulier écossais, et irlandais de souche écossaise qui s'installèrent dans le massif des Appalaches et dans les Monts Ozarks à partir du XVIII siècle. Émigrés en provenance directe d'Europe, ou métayers fuyant les conditions de travail inhumaines des plantations de Virginie, de Caroline et de Géorgie, ces colons apportèrent avec eux une tradition musicale très vivace, faite de ballades à caractère narratif, de chansons dites lyriques, plus intimistes, de comptines, de cantiques, d'hymnes et de psaumes, tels ceux des 
églises Baptistes et d'airs de danse, la plupart pour violon. II existe également des formes inclassables ou hybrides, entre airs de danse et ballades.

A la fin du XIXe siècle, le musicologue américain Francis Child, de l'Université de Harvard, collecta dans les îles Britanniques plus de trois cents ballades traditionnelles. Son homologue britannique Cecil Sharp retrouva la trace d'une centaine d'entre elles sur le territoire américain, perpétuées par les fermiers des Appalaches et des Ozarks. Ces ballades et chansons lyriques, abordent fréquemment des thèmes tragiques (amours malheureuses, adultères, abandons, meurtres et suicides), la mort violente étant la conclusion la plus commune d'une vie difficile. Dans un registre plus gai, de nombreuses chansons s'attachent à décrirent des catastrophes spectaculaires ou les exploits de criminels célèbres².

Cette tradition musicale connut en Amérique une double évolution. L'isolement imposé par la distance entre les fermes et les villages, un relief escarpé et des conditions climatiques difficiles, en renforça l'enracinement local ; les textes furent adpatés à des lieux, des individus ou des événements régionaux. Mais sur le sol américain, ces musiques rencontrèrent d'autres traditions : les idiomes des tribus indiennes, ceux des esclaves noirs importés pour suppléer à la main-d'œuvre celtique rebelle, ou encore le yodel, les valses et les polkas des immigrants slaves et germaniques venus exploiter, au début du XXe siècle, les gisements de charbon, à quoi il faut ajouter quelques mesures de musique italienne, mexicaine ou française (cajun) et les airs de music-hall et de gospel écrits par des professionnels de la côte Est. Ainsi, à côté d'instruments propres à la communauté blanche appalachienne, tel que le dulcimer (une caisse de résonance ovale montée avec quatre cordes que l'on joue posée à plat sur les genoux), ou l'autoharp (une petite harpe équipée de mécanismes qui en étouffant certaines cordes, permettent de jouer automatiquement des accords) viennent s'ajouter la mandoline des italiens, la guitare, vraisemblablement rapportée de la guerre de Cuba (1898) par les soldats noirs et le banjo, adaptation américaine d'instruments africains.

Progressivement, d'autres formes de musique rurale apparaissent : les cowboys songs des années 1930, qui profitent de l'engouement que suscite l'exploitation hollywoodienne de l'esprit de la frontière 3 . Au Texas, Bob Wills a l'idée d'incorporer les influences du blues et l'esprit du swing, style de jazz alors extrêmement populaire, à la musique rurale, donnant naissance au western swing, forme sophistiquée et irrésistiblement dansante. Preuve du succès de ce style, la musique rurale va pendant quelques décennies s'appeler Country \& Western. Avec la guerre, une nouvelle forme de country apparaît, où sur des rythmes marqués et des sonorités électrique est décrite avec réalisme la vie quotidienne des petites gens. On l'appelle honky tonk, du nom des petits bars où se retrouvent travailleurs 
agricoles, chauffeurs routiers et ouvriers, bars où le sexe et l'alcool font bon ménage4.

Au début des années 1960, la musique country est solidement implantée à Nashville et la ville s'installe dans l'imaginaire de tout le pays lorsqu'à partir de 1964, l'émission Grand Ole Opry est retransmise à la télévision. De nombreuses compagnies de disques nationales y installent une succursale (dans le célèbre quartier de Music Row) et y ouvrent des studios d'enregistrement. La ville attire un nombre croissant de musiciens et de producteurs et donne naissance à un style de country spécifique, de plus en plus éloigné des sons rustiques des premiers temps de la country. Progressivement, toute l'économie de la ville repose sur l'industrie musicale ; plus de la moitié des disques produits aux États-Unis y sont enregistrés. Mais le succès de la musique rock met en danger l'establishment musical de Nashville. Celui-ci contre-attaque par l'intermédiaire du guitariste virtuose Chet Atkins qui met progressivement au point un son, le Nashville Sound, destiné à rivaliser avec les sonorités pop à la mode (on parlera justement de "country pop"), à base de violons, de chœurs féminins et de guitares dites "pedal-steel" (aux sonorités hawaïennes). Cette country-là n'est plus la musique d'une région mais plutôt d'une classe, la classe ouvrière urbaine ou rurale ; comme elle, elle affiche volontiers des positions conservatrices, alors que paradoxalement, le milieu d'où elle a jailli (la mine ou la ferme), était politiquement et socialement plus ouvert. Dans cette nouvelle country, la part de l'héritage appalachien diminue. On ne le retrouve que dans le bluegrass, style incarné depuis la fin des années 1940 par Bill Monroe, Flatt \& Scruggs, Doc Watson et Ralph Stanley, qui séduit un public souvent jeune, féminin et intellectuel, déçu des fadeurs prévisibles du Nashville Sound. Le bluegrass est rapide, dansant, on y entend des échos de ragtime, de blues et de jazz, et les instruments, tous acoustiques, rivalisent de virtuosité. Depuis les années soixante, d'autres artistes sont apparus, dont un nombre croissant de femmes (à l'exemple de Lucinda Williams, Gillian Welch, Rosanne Cash ou Emmylou Harris ). Ils définissent une country plus innovatrice (country rock ou progressive country) qui incorpore l'instrumentation électrique et le rythme du rock à des thématiques typiquement country ; s'y rattache l'école d'Austin (outlaw country), emmenée par Willie Nelson et Waylon Jennings.

La musique country est dans son ensemble une composante essentielle de l'industrie du disque américaine. En septembre 2004, 10 albums de country étaient classés parmi les 50 meilleures ventes de disques aux Etats-Unis (certains, comme celui de Kenny Chesney, depuis 40 semaines). Sur ces 10 albums, 4 se sont hissés à la première place des ventes, tous styles confondus, l'un d'eux s'y maintenant 10 semaines (Tim McGraw). Si l'on s'intéresse aux chiffres de ventes cumulés (et non 
ceux d'une seule semaine ou d'un seul mois), sur les 14 artistes ayant atteint le niveau "platine" en septembre 2004 ( 1 million ou plus d'albums vendus), 6 étaient des artistes country, avec pour trois d'entre eux, des ventes s'élevant à 4, 3 et 2 millions d'albums (Toby Keith, Kenny Chesney et Sara Evans). Sur les 20 meilleures ventes country du même mois, 12 avaient dépassé le million d'exemplaires. Le total des ventes pour l'année 2003 montre que la country se classe en troisième position, avec 69 millions d'albums vendus, après le rhythm \& blues (195 millions) et le rock alternatif (131 millions), mais avant le rap (67), le rock "metal" (66), le rock "chrétien" et le gospel (50), les musiques de films (40), le jazz (20), la musique classique (16), le style "latin" et la new age. C'est par ailleurs le seul style dont les ventes augmentèrent (de 12\%) au cours de l'année 2003. Malgré sa relative discrétion sur le marché international, les chiffres publiés par le magazine professionnel Billboard confirment que la country est devenue force majeure sur le marché intérieur.

L'histoire de cette musique, qui ne peut se réduire à une succession de styles où le dernier venu éclipserait le précédent, est jalonnée de retours en arrière et de redécouvertes. Elle est actuellement en train de connaître la mutation la plus importante des vingt dernières années, mutation sous forme de renaissance. Le point de départ de cette renaissance, c'est la bande sonore originale (B.O.) du film O Brother, Where Art Thou ? des frères Ethan \& Joel Coen. Le film, inspiré de l'Odyssée, met en scène dans l'Amérique des années 1930 les aventures de trois détenus blancs qui deviennent des stars de la musique country. La musique du film est parue en 2000 chez Mercury Records. II s'agit d'une compilation de morceaux acoustiques, certains dans leur version originale, d'autres interprétés par des artistes contemporains. On y entend "Po' Lazarus," work-song chanté par un quatuor de prisonniers qu'Alan Lomax a enregistré en 1959 dans une ferme prison du Mississippi, "Big Rock Candy Mountain," rêve hobo interprété par son auteur, Harry Mc Clintock, en 1928, "In the Jailhouse Now," un classique country écrit la même année par Jimmie Rodgers, et "You Are My Sunshine," composition de Jimmie Davis, qui sera deux fois gouverneur de la Louisiane, interprétée ici par Norman Blake, sept gospels, dont "Down To The River To Pray," chanté par la plus grande artiste actuelle de bluegrass, Alison Krauss, rejointe pour "I'll Fly Away" par Gillian Welch, autre star de bluegrass, "Keep On the Sunny Side," popularisé dans les années trente par la célèbre Carter Family et repris ici par une autre famille de musiciens bluegrass, les Whites, "In The Highways" par les sœurs Peasall et "I Am Weary (Let Me Rest)" par la famille Cox, également issue du milieu bluegrass, un blues, "Hard Time Killing Floor Blues" de Skip James chanté par Chris Thomas King, le principal acteur noir du film, "Didn't Leave Nobody But The Baby," field holler interprété par Alison Krauss, Gillian Welch, et Emmylou Harris, enfin une 
complainte élégiaque, "O Death," par Ralph Stanley, un des créateurs du bluegrass. Nulle trace, nulle influence du Nashville Sound, qui est pourtant le style de country toujours le plus écouté aux Etats-Unis, dans cette compilation, qu'il s'agisse des auteurs, des compositeurs ou des interprètes.

Or, la B.O. de $O$ Brother, plusieurs semaines $n^{\circ} 1$ des ventes, tous styles confondus, s'est vendue à 7 millions d'exemplaires, a obtenu en 2002 cinq récompenses à la cérémonie des Grammy, dont la plus prestigieuse, "album de l'année." De plus, elle est à l'origine d'un spectaculaire regain d'intérêt pour ces musiques "archaïques". Pour preuve, le succès des nombreuses compilations vendue sous l'étiquette americana, du concert avec les musiciens de la B.O. au célèbre Ryman Auditorium de Nashville, concert qui a fait l'objet d'un disque et d'un film et des tournées à guichets fermés qui se multiplient dans toutes les régions des Etats-Unis, attirant un public de tous âges, sans aucun soutien de la part des radios ni des télévisions5. II s'agit donc bien parler d'une renaissance, d'un revival de la musique rurale traditionnelle du sud.

Ces remarques appellent à la fois commentaires et développements. Que pouvonsnous en déduire quant aux raisons profondes du succès de cette compilation et du regain de popularité des musiques rurales traditionnelles ? Quel sens donner à ce revival ? Pour comprendre le succès de ce disque, il faut d'abord insister sur celui du film, dans la mesure où il a permis à des millions de spectateurs d'être facilement mis en contact avec ces musiques, dans le confort et la sécurité d'une salle de cinéma ou de leur salon, sans l'effort que nécessite le déplacement à un concert. D'autre part, il faut souligner que plus qu'une simple bande originale, ce disque est en fait une anthologie, un recueil de grands succès, de classiques qui font partie du patrimoine culturel commun à de nombreuses familles américaines, transmis de génération en génération, et qui n'avait pas pour autant encore fait l'objet de multiples rééditions phonographiques ou d'une surexposition médiatique qui en auraient tué la fraîcheur. Enfin, ces musiques utilisent des modes ancestraux, en particulier le mixolydien, et des gammes pentatoniques, que certaines formes de rock, de jazz et de world music ont remis au goût du jour, ce qui confère à la musique rurale traditionnelle une modernité dont les formes plus récentes de country ne bénéficient pas.

II est intéressant d'observer les termes qu'utilisent sur les livrets des différents disques d'old-time music (voir discographie) des journalistes musicaux ou des artistes comme Jay Orr, les frères Coen, John Currie, Fred Jasper, Sheila Key Adams ou Marty Godbey pour qualifier ces musiques et essayer d'en expliquer le soudain et imprévu retour en grâce. On note tout d'abord des remarques portant sur la technique musicale : "raw, acoustic simplicity... quiet, spare instrumentation... home-made music... front-porch feel... humble beginnings...", remarques qui 
soulignent l'aspect artisanal et acoustique, familial ou communautaire de ces musiques (certains parlent d'ailleurs de "community feeelings" à leur écoute6). De telles caractéristiques sont censées être représentatives d'une attitude anticommerciale, ce qui est connotée positivement. Ce sont des critères similaires qui poussèrent les militants socialistes et communistes des années 1930 et 1940 à choisir le folk pour accompagner leurs luttes. Le dépouillement sonore et l'absence d'instruments électriques sont importants ; c'est ce qui permet de faire la différence entre ce que certains appellent "the stale, the choking gas of packaged, processed, radio fare" et les musiques qui apportent "a musical oxygen7". L'espace scénique lors du concert au Ryman Auditorium, avec son éclairage discret et sa scène nue, sans enceintes de retour ni amplificateurs envahissants, confirme cette lecture. Nous retrouvons ici l'opposition classique entre culture folk aux sons acoustiques d'une part, et culture de masse (ou industrielle) aux sons électriques de l'autre, la première étant pure et spontanée, la deuxième "produite" et donc aliénante. Pourtant, comme le rappelle Lawrence Grossberg, "there are no pure cultural practices, as if records with acoustic guitars were somehow less contaminated by the technologies and economies of capitalist culture...Nor is there ever a simple relation between specific cultural practices and preconstituted social groups which exist independently of their cultural practices" (77).

Ces sons acoustiques, qui résultaient autrefois d'une contrainte technique, sont désormais un choix esthétique délibéré. Difficile de dire ce qu'un Jimmie Rodgers ou un Robert Johnson joueraient aujourd'hui. Nous savons cependant que dès que les musiciens de country ou de blues rural en ont eu la possibilité, ils ont électrifié leurs instruments, provoquant souvent la colère des tenants de l'orthodoxie (en témoignent l'accueil réservé à Ernest Tubb au Grand Ole Opry ou plus tard à Bob Dylan à Newport). Dans les années 1960, certains bluesmen, comme Big Bill Broonzy, passés à l'électricité furent même obligés lorsque le circuit folk les a redécouverts sur de vieux disques, de reprendre la guitare acoustique de leurs débuts. Dans le cas des musiques acoustiques de la B.O. d'O Brother, le fait même que ces musiques soient encore jouées aujourd'hui témoignent, aux yeux des critiques, de leur force, de leur qualité, et par là, de leur supériorité sur les musiques contemporaines, dont ils supposent qu'elles ne passeront pas l'épreuve du temps. La renvendication d'un retour au passé n'est pourtant pas sans risques. Imanuel Wallerstein souligne l'ambivalence de toute forme de renaissance. En revendiquant "une période antérieure de gloire culturelle" on reconnait en même temps "l'infériorité culturelle du moment" (85).

Se dessine alors une correspondance entre des critères purement techniques et des valeurs morales, correspondance soulignée par le recours à des termes abstraits : "dignity, humanity, sincerity, purity, honesty," ou même "decency" 
(Cantwell 14). Une telle alliance de morale et d'esthétisme révèle chez les auteurs de ces textes une tendance à attribuer un sens à une forme. Ce n'est que par un raccourci un peu simpliste qu'il leur est possible de relier des caractéristiques musicales au sens éthique des générations précédentes, sens qu'ils imaginent plus développé que le leur. On ne s'étonnera pas, par ailleurs, que dans des cultures ouvrières urbaines ou rurales marquées par le conservatisme, ces remarques à valeur morale côtoient des termes empruntés au vocabulaire religieux. C'est ainsi que dans ses notes pour l'albumDown From the Mountain, Jay Orr parle de "résurrection" à propos du regain de popularité dont bénéficient ces formes musicales (de nombreuses chansons ont d'ailleurs pour thème des résurrections), les concerts ayant lieu, ajoute-t-il, devant des "publics convertis8". Le fond de scène du Ryman Auditorium, ancien temple protestant reconverti en salle de concert, représente d'ailleurs des fenêtres d'église en ogive. On remarque également dans ces choix lexicaux l'importance de la notion d'authenticité, à l'origine de débats parfois virulents entre les artistes et leurs publics. Le terme revient fréquemment chez les historiens de la musique populaire. Daniel Kingsman par exemple affirme : "bluegrass has maintained the authenticity and freshness of a traditional style" (194) ; Robert Cantwell décrit même le bluegrass comme une façon de "ré-authentifier et traditionnaliser" la musique hillbilly (151). Pour des raisons complexes (conservatisme, nostalgie de la jeunesse, sentiment d'appartenance à une communauté, à un groupe de pairs), publics et critiques semblent attacher beaucoup d'importance à ce que les artistes qu'ils ont choisis demeurent authentiques, autrement dit, fidèles au modèle original, qu'ils ne trahissent pas les communautés qui les soutiennent, quitte à ce que cette authenticité soit, paradoxalement, recréée. C'est ainsi que les frères Coen ont insisté pour que quelques chansons contemporaines soient inclues lors du concert Down From the Mountain parce qu'elles avaient "the feel of vintage authenticity9." Par souci d'authenticité, certains se tournent alors vers la musique de cultures minoritaires, opprimées pour des raisons ethniques, culturelles ou économiques, ici les petits fermiers blancs des Appalaches et du Sud, musique qui aurait à leur yeux préservé un degré d'authenticité supérieure. Plus que des critères stylistiques ou des caractéristiques musicales, c'est donc le statut de ses pratiquants qui souvent confère à une musique donnée son aura d'authenticité.

Avant tout, ces musiques représentent "a deliberate reconstruction of a Southern past," comme l'écrit Robert Cantwell (151). Cette formulation est importante ; elle souligne l'ancrage à la fois géographique et temporel de ces musiques. Ces musiques sont effectivement celles d'un territoire, le Sud, des vallées septentrionales des Appalaches au Golfe du Mexique. Comme toute forme musicale, elles en délimitent avec force et précision les contours10. Elles 
correspondent à un imaginaire d'autant plus chargé mythiquement qu'il renvoie à des territoires et à une culture longtemps méprisés et tournés en dérision, parfois sources d'une vague inquiétude, comme le suggère le film Délivrance de John Boorman (1972). Quelques productions culturelles populaires, plus rares qu'on pourrait le penser, tels les romans de Lee Smith ou les films de Clint Eastwood ont contribué à donner une image moins primitive et plus positive du Sud. Ces territoires évoquent une Amérique rurale, jeffersonienne, pré-industrielle, pré-lapsarienne presque (la chute étant peut-être ici, plus que l'industrialisation, la défaite lors de la Guerre de Sécession), à une époque de mutation de l'environnement, où l'urbanisation inquiète plus qu'elle ne séduit, où la notion de progrès est devenu une plaisanterie amère, où la mondialisation de l'économie capitaliste ne suscite plus guère d'espoirs. Comme l'écrit Larry Portis à propos du minstrelsy, forme musicale antérieure à la country, "new urban people experienced more of a loss of identity than any acquisition of new attachment. From this point of view, minstrelsy expressed the existential angst of a collection of individuals who were losing their cultural and, often, emotional bearings"(28). Le revival déclenché par $O$ Brother serait-il du même ordre ? Le territoire de $O$ Brother, sauvage et isolé, ce que Cantwell appelle "the edenic Old South and boundless West" (xii), fait écho, dans sa rudesse même, aux premiers temps de la colonisation, aux origines difficiles mais idéalisées du pays.

La dimension spatiale se double alors d'un ancrage temporel, signe distinctif de tout revival. Les racines européennes de la country appalachienne l'inscrivent dans une tradition qui remonte au moins jusqu'au XVe siècle. Les artistes qui ont pu être enregistrés dans les années 1960 étaient déjà âgés. Leur âge et celui de leur musique fait partie de l'attirance qu'ils exercent ; ils rassurent et permettent de renouer, sur le mode nostalgique, avec un passé idéalisé, plus fabuleux qu'historique, qui offre une image de stabilité et de permanence, contre-poids à l'accélération de la société américaine. C'est ainsi que dans la presse abondent des épithètes telles que "timeless," "immortal," "enduring" ou "eternal" et que les notes de pochette de $O$ Sister! parlent de "vintage music...when life was simpler." Abrahams souligne la dimension rassurante du retour à un passé rural : "Urban man's major anxieties arise from lack of tradition, restlessness, constant state of change. His solution might be a clinging to vestige of peasant or primitive values, e.g. pastoralism" (11).

Enfin, le terme de "reconstruction" utilisé par Cantwell dans la citation citée précédemment rappelle qu'il ne s'agit pas de la simple reproduction de l'original. Tout revival implique en effet une réinterprétation du passé. C'était déjà le cas pour le premier revival des musiques rurales, celui des années 1930, qui passe souvent inaperçu. De fait, comme c'était la première fois que ces musiques étaient 
enregistrées et passaient à la radio (médium qui joua un rôle essentiel dans la propagation et le succès de la musique country), elles furent commercialisées comme étant la captation fidèle de la tradition. Mais ces premiers disques étaient déjà des reconstructions des musiques du XIXe siècle modifiées pour plaire au public de l'époque par l'adjonction d'influences western, jazzy, hawaïenne ou d'emprunts au music-hall. Un deuxième revival eut lieu à la fin des années 1950, en parallèle avec celui du blues. Cette deuxième renaissance se développa dans les états du Nord au sein du milieu étudiant libéral et sa dimension politique transforma à son tour le son et l'esprit des œuvres traditionnelles utilisées. La B.O. de $O$ Brother, qui est donc à l'origine d'un troisième revival, est elle-même adaptée aux exigences contemporaines : peu de morceaux a cappella, pourtant majoritaires au XIXe siècle, amélioration substantielle de la qualité sonore, ré-enregistrement par des artistes contemporains, etc.

Les connotations morales et religieuses ( 7 gospels figurent sur le disque) révèlent une quête de repères et de valeurs que le 11 septembre n'a pu qu'intensifier. C'est d'ailleurs à une autre époque de crise, celle des années 1930, qu'a eu lieu le premier revival country, tandis que la deuxième vague, plus intellectuelle, participait de l'inquiétude que certains pouvaient éprouver au cours des années 1950 face à la montée de l'urbanisation et de l'industrialisation, aux nouvelles orientations en politique étrangère et à la traque de la différence et de la dissidence à l'intérieur du pays. En marquant aujourd'hui leur intérêt pour ces musiques, ceux que le nationalisme outrancier et le néo-impérialisme actuels gênent peuvent néanmoins faire preuve de leur attachement à leur pays, à ses racines culturelles, à des valeurs morales traditionnelles. Le goût pour les paisibles ou tragiques chansons acoustiques du passé représente, plus qu'un choix esthétique, l'incarnation sonore d'un état d'esprit. Une dernière explication mérite attention, celle avancée par Cantwell selon laquelle le départ des sudistes pour le nord ou la Californie a été vécu comme l'expulsion de l'Eden : la musique servirait alors à reconstruire cet Eden en recréant un contexte à la fois géographique, temporel et religieux (48). Les musiques du disque, fortement marquées par leurs racines anglo-irlandaises, offrent de surcroît l'impression trompeuse d'être un idiome parfaitement blanc et spécifiquement conçu pour la communauté blanche. Derrière ce cliché tenace, la réalité est bien plus complexe.

La country, loin d'être une musique non métissée et destinée exclusivement à un public de fermiers blancs traditionalistes, les rednecks, a été très tôt adoptée par de nombreux musiciens noirs. A l'inverse, les plus grands artistes blancs de country ont été nourris par les sonorités et les techniques afro-américaines. Quelques exemples : le célèbre style de guitare de Maybelle Carter (le "Carter lick"), dont de nombreux musiciens continuent de s'inspirer, lui a été enseigné par son ami Lesley 
Riddles, un Africain-Américain, Jimmie Rodgers a appris à jouer de la guitare avec un voisin noir, avant d'enregistrer avec Louis Armstrong, Clifford Gibson (un bluesman de St. Louis) ou des Mexicains, Jimmie Davis s'est produit avec Oscar Woods, Al Dexter avec son "Colored Band" (qui comprenait Claude Crawford et Lee Siegler), Bill Monroe a fait son apprentissage avec Arnold Shultz, un ami noir, Doc Boggs avec les mineurs noirs de sa région, Roscoe Holcomb en écoutant les disques du bluesman Blind Lemon Jefferson... II est par ailleurs arrivé que des enregistrements de country blues blanc soient étiquetés "race" (noirs) pour des raisons commerciales, tandis que Chuck Berry, sur la foi des sonorités country de son premier succès, "Maybellene", passait souvent pour un blanc. Quant à Elvis, de nombreuses radios refusèrent dans les premiers temps de passer ses disques, croyant avoir affaire à un chanteur afro-américain.

Il faut également noter que le stéréotype qui fait de la country une musique primaire, écrite par et pour des machos, ne résiste guère à l'analyse. Les musiques folk étaient déjà un domaine de prédilection pour les femmes (comme l'atteste le roman historique The Devil's Dream, de Lee Smith). Certaines ont d'ailleurs retrouvé une deuxième carrière au cours des années soixante (Almeda Riddle, Cousin Emmy, Sarah Ogan Gunning). L'arrivée du disque a vu l'éclosion du talent de la famille Carter, et les personnalités les plus fortes, les réussites les plus éclatantes s'observent bien souvent du côté des artistes féminins. La liste en est longue, mais nous pouvons néanmoins citer les noms de Rose Maddox et Patsy Montana, puis, après guerre, de Dolly Parton, Patsy Cline, Loretta Lynn, ou Patty Loveless, et aujourd'hui des Dixie Chicks, Alison Krauss, Shawn Colvin et Shelby Lynne.

$\mathrm{Si}$ l'ancrage spatial et temporel des musiques de $O$ Brother ne fait pas de doute, c'est en revanche de déterritorialisation qu'il faudrait parler pour qualifier son inscription sociale. II est en effet très difficile de ramener le succès de ces musiques aux goûts d'une classe précise. La country de Nashville, qui s'est longtemps adressée essentiellement à un prolétariat urbain et rural sudiste s'est aujourd'hui infiltrée dans les résidences plus cossues des classes moyennes des banlieues du nord. Mais l'old-time music bouscule encore plus les idées reçues. La B.O. de $O$ Brother, tout en se distinguant du strass et des paillettes de Nashville et de ses stars réactionnaires, n'en exclut pas pour autant le public. S'y retrouvent en effet, à côté d'un public intellectuel, souvent jeune et féminin, ouvert culturellement et socialement, un public tout autre, ouvriers, mineurs, chauffeurs routiers, secrétaires, commerçants et employés de l'Amérique blanche des campagnes, des petites villes et des banlieues qui y entendent un écho de leurs tragédies, de leurs espoirs, de leurs vies.

II faut en conclusion s'interroger sur la portée de cette renaissance de lold-time music. Dans quelle mesure s'agit-il d'un simple mouvement de mode, cyclique et 
passager, un effet de nouveauté, au bout du compte marginal, ne modifiant pas le mainstream en profondeur ? Tout dépend de l'attitude des radios. Celles-ci n'existent que par et pour la publicité, la musique n'étant qu'un moyen plutôt indolore et relativement efficace de diffuser les annonces commerciales qui sont leur raison d'être. II leur faut alors attirer les publics les plus rentables, ceux susceptibles d'acheter les produits de leurs annonceurs, c'est-à-dire, au niveau national, la ménagère de classe moyenne ; la musique passée sur les ondes doit lui correspondre. Cela exclut les programmations hasardeuses, aléatoires ou risquées. Les DJ locaux n'ont plus guère leur mot à dire, ce sont les "formats" décidés au niveau national qui s'imposent. Quatre ans après le succès commercial inattendu de la B.O. du film, ces formats excluent encore les musiques qui l'ont inspirée, jugées trop crues ou trop pessimistes pour la ménagère américaine. Pourtant, la concurrence d'Internet et des radios spécialisées par satellite, telles America, Nashville!, X Country, Hank's Place, Bluegrass Junction ou The Village a changé la donne. Des marchés plus petits, fractionnés, sont devenus rentables pour les annonceurs, des goûts musicaux plus éclectiques deviennent légitimes. Alors, à côté de la country édulcorée du Nashville Sound, les radios se décident timidement à jouer les musiques rurales traditionnelles, condition essentielle pour prolonger le revival qu'elles connaissent.

Interrogé à ce sujet, John Rumble, historien en chef du Country Music Museum and Hall of Fame de Nashville fait preuve d'optimiste. Ces musiques, explique-t-il, n'ont jamais vraiment disparu, c'est une question de visibilité, de mise en valeur. Nashville n'est pas un monstre, un "villain," c'est avant tout une entreprise commerciale, et aucune musique n'est a priori exclue si elle s'avère rentable. De surcroît, les différences entre styles doivent être relativisées. II existe des caractéristiques communes à toutes les formes de country (par exemple une certaine dimension spirituelle, ou humaniste, et leur simplicité) qui devrait confirmer la place grandissante qu'occupent les musiques traditionnelles dans les programmes de radio. En définitive, le risque le plus grave pourrait être celui d'une surexposition. Certes, depuis le premier revival, l'impératif commercial a toujours été présent, comme le souligne Robert Cantwell : "Hillbilly music has never been anything but entrepreneurial and commercial, prospering in the commodity which in America is never in short supply -the past"(13). Mais la mise sur le marché à un rythme soutenu de compilations et de rééditions d'old-time music et des campagnes de publicité trop fréquentes risquent de tuer le succès de musiques qui symbolisent précisément le refus du marketing agressif et de la massification. 


\section{Notes}

Merci à John Rumble, Chief Historian au Country Music Museum \& Hall of Fame de Nashville, pour ses précieux commentaires et ses suggestions éclairantes.

1 On retrouvera ce terme après la deuxième guerre mondiale, modifié en rockabilly, pour désigner la rencontre de la musique country avec le rhythm \& blues noir.

2 Parmi les plus représentatives, on citera "Fatal Flower Garden" (interprétée par Nelstone's Hawaiians), "The Butchers Boy" (Buell Kazee), "When That Great Ship Went Down" (William and Versey Smith) ou encore "Engine One-Forty-Three" par la Carter Family.

3 La petite centaine d'authentiques chansons de cowboys et la poignée de véritables garçons vachers qui les interprètent ne furent pas suffisants. Une nouvelle génération de chanteurs n'ayant qu'un lointain rapport avec le métier de cowboy ou même de fermier, popularisa alors un style hybride où des chansons de variété, de vaudeville ou même de jazz prirent une coloration cowboy ; on y rencontre, habillés en style western, Tex Riter, Gene Autry, Roy Rogers ou Patsy Montana chantant "I Want to be a Cowboy's Sweetheart" ou encore "Home on the Range".

4 Des artistes comme Ernest Tubb, Hank Williams, Hank Snow, Pee Wee King, l'auteur de "Tennessee Waltz", Roy Acuff, le compositeur de "Jole Blon" (jolie blonde, en Cajun), Lefty Frizzell, Webb Pierce ou Patty Loveless popularisèrent le style honky-tonk en abordant des thèmes désenchantés, moins religieux ou nostalgiques qu'autrefois, peignant avec un mélange de sentimentalité et de réalisme parfois extrêmement cru les amours malheureuses, la violence, l'alcool, l'adultère ou la prostitution.

5 II y a même une anthologie de chanteuses de bluegrass intitulée, comme c'est original, O Sister, déjà au volume II (voir discographie)

6 Notes de pochette de Songcatcher II.

7 Notes de pochette de Down From the Mountain.

8 Cantwell y voit là une forme dérivée des revivals religieux, 38 .

9 Notes de pochette de la B.O. du film O Brother.

10 Je renvoie ici au passage éclairant de Mille Plateaux où Deleuze et Guattari attribuent à la musique une énorme capacité de territorialisation, 311. 


\section{Bibliographie}

ABRAHAMS, Roger D., \& George Foss. Anglo-American Folksong Style.. Englewood Cliffs: Prentice-Hall, 1968.

CANTWELL, Robert. The Bluegrass Breakdown, the Making of the Old Southern Sound. New York: Da Capo, 1984.

---. When We Were Good: The Folk Revival. Cambridge, MA: Harvard University, 1996.

CHILD, Francis James. The English and Scottish Popular Ballads (1882-1898). New York: Dover , 1965.

COFFIN, Tristram. The British Traditional Ballad in North America. Philadelphia: America Folklore Society, 1950.

DELEUZE, Gilles \& Félix Guattari. Mille plateaux.Capitalisme et schizophrénie 2. Paris : Minuit, 1980.

FORD, Ira. Traditional Music of America. New York: E. P. Dutton \& Co., 1940.

GENTRY, Linnell, ed. A History and Encyclopedia of Country, Western and Gospel Music. Nashville: Clairmont Corp., 1969.

GREEN, Douglas B. Country Roots. New York: Hawthorne, 1976.

GROSSBERG, Lawrence. We Gotta Get Out of This PLace. Popular Conservatism and Postmodern Culture. New York : Routledge, 1992.

GURALNICK, Peter. Lost Highway. New York: Vintage, 1982.

---. Feel Like I'm Going Home (1972). New York: HarperCollins, 1994.

HERZHAFT, Gérard. La Country Music. Paris : PUF-Que Sais-je? 1975.

KINGSMAN, Daniel. American Music, A Panorama. New York: Schirmer, 1979.

KINGSBURY, Paul \& Alan Axelrod, eds. Country: The Music and the Musicians. New York: Abbeville, 1988.

LOMAX, Alan. Folk Songs Style and Culture. New Brunswick: Transaction, 1968.

LOMAX, John A. Cowboy Songs and Other Frontier Ballads. New York: Macmillan Co., 1916.

LYNN, Loretta, with George Vecsey. Coal Miner's Daughter. New York: Da Capo, 1988.

MALONE, Bill \& Judith McCulloh, eds. The Stars of Country Music. Urbana, IL: University of Illinois, 1975.

---. Country Music U.S.A.. Austin, TX: University of Texas, 1985.

MARCUS, Greil. Invisible Republic, Bob Dylan's Basement Tapes. New York: Henry Holt, 1997.

MAYER, M. Margaret \& Claude Chastagner. The American Dream, American Popular Music. Santa Barbara: The Front Desk, 1994. 
MILES, Emma Bell. The Spirit of the Mountains (1905). Knoxville, TN: University of Tennessee, 1975.

PORTIS, Larry. Soul Trains, A People's History of Popular Music in theUnited States and Britain. College Station, TX: Virtual Bookworm.com., 2002.

RANDOLPH, Vance \& Floyd C. Shoemaker.Ozark Folksongs. Columbia, MO: The State Historical Society of Missouri, 1946-1950.

RITCHIE, Jean. The Singing Family of the Cumberlands. Louisville: The University Press of Kentucky, 1988.

ROSENBERG, Neil V. Bluegrass, A History. Urbana, IL: University of Illinois Press, 1985.

ROSENBERG, Neil V., ed. Transforming tradition: Folk Music Revivals Examined. Urbana, IL: University of Illinois, 1993.

SHARP, Cecil. English Folk-Songs from the Southern Appalachians. Maude Karpeles ed. London: Oxford University, 1932.

TOSCHES, Nick. Country, The Twisted Roots of Rock'n'Roll. New York: Da Capo, 1977.

---. Unsung Heroes of Rock'n'Roll. New York: Charles Scribner's Sons, 1984.

WALLERSTEIN, Immanuel. Le Capitalisme historique. Paris: La Découverte, 1985. 


\section{Discographie}

Music from the Motion Picture "O Brother, Where Art Thou?" Mercury Records, 2000.

O Sister!, The Women's Bluegrass Collection (vol 1 \& 2) Rounder, 2001 \& 2002.

Down From the Mountain. Mo Zoss Records, 2001.

Songcatcher II. Vanguard Records, 2002.

Appalachian Stomp, Bluegrass Classics. Rhino, 1995.

Anthology of American Folk Music edited by Harry Smith, Smithsonian Folkways, 1997.

les excellentes compilations éditées par Frémaux \& Associés:

Folksongs

Old Time Country Music USA, 1926-1944

Country, Nashville, Dallas, Hollywood. 1927-1942

Country, Vol 2, Gospel, New York, Atlanta, Nashville, 1929 -1946

Hillbilly Blues, 1928-1946

Country Boogie, 1939-1947

Country Music, Changing Times, Bluegrass, Honky Tonk, West Coast \& Western Swing, 1940-1948

Western, Cowboy Ballads \& Songs, 1925-1939

Western Swing, Texas, 1928-1944

Guitar Country, 1926-1950

et les nombreux documents historiques de la collection Folkways, dont le catalogue est disponible à l'adresse suivante :

Smithsonian Folkways Recordings

955 L'Enfant Plaza, Suite 7300

Washington, DC 20560-0953 USA 